\title{
Three-Dimensional Displacement and Strain Measurements by Windowed Phase-Shifting Digital Holographic Interferometry
}

\author{
Yoshiharu Morimoto and Motoharu Fujigaki \\ Moire Institute Inc. E Wakayama University \\ Japan
}

\section{Introduction}

It is important to measure displacement and strain distributions to prevent failures of structures. However, one point measurement method such as strain gage is widely used in industrial practice. It is time-consuming and difficult for one-point method to find the point with the maximum strain on the structures. Whole-field optical methods such as moire method, digital image correlation, speckle method and holography are effective to find the point with the maximum strain (Sharpe, 2008). Especially, holography provides three dimensional displacement information and high resolution.

In conventional holography, an object beam and a reference beam interfere each other on a high-resolution photographic plate. It is necessary to develop the photographic plate (hologram) and it is time-consuming and bothersome work. In conventional holographic interferometry, holograms recorded before and after deformation are superposed on a photographic plate and the displacement is obtained from the interference fringe pattern (Valery et al., 1996; Ranson et al., 1993; Hayashi et al. 1986). In digital holography, a hologram is usually recorded on a CCD or C-MOS sensor instead of a photographic plate (Yaroslavskii et al., 1980; Pedriniet al., 1998; Schnars, et al. 2005). In phase-shifting digital holographic interferometry (PSDHI), the complex amplitude of the object is analyzed from the phase-shifted holograms obtained by shifting the phase of the reference beam. The reconstructed image can be calculated from the complex amplitudes of the hologram using the Fresnel diffraction integral with a computer (Yamaguchi et al., 1997; Zhang et al., 1998). The displacement at each point of an object is obtained from the phase-difference between the reconstructed images analyzed from the digital holograms recorded before and after deformation. The equipment is simple and the analysis is fast. It is useful for practical field measurement of displacement and strain distributions.

Holograms and reconstructed images have speckle noise and they provide large error in the calculation of displacement and strain analysis. In order to reduce the effect of speckle noise, the authors developed a novel method, i.e., the windowed phase-shifting digital holographic interferometry (Windowed PSDHI). In holography, any part of a hologram has the optical information about the whole reconstructed image. By using this feature of 
holograms, a hologram is divided into several parts by superposing many different windows. The phase-difference values at the same reconstructed point obtained from any other different part of the hologram should be the same. If there is speckle noise, the phase-difference with higher intensity at a reconstructed point is more reliable than that with lower intensity. Therefore, the phase-difference at each point is selected when the intensity is the largest at the same point (Morimoto et al. 2004, 2005a), or the phasedifference is calculated by averaging the phase-differences obtained from all the windowed holograms by considering the weight of the intensity (Morimoto et al. 2005b, 2005c, 2007).

In this study, in order to check the effect of the number $n$ of the windowed holograms on accuracy, the number $n$ is changed. The weight for averaging is also changed. When the weight is the $m$-th power of the absolute amplitude of the complex amplitude of the reconstructed object, the accuracy, that is, the standard deviation of the analyzed displacement error changes according to the power $m$. The optimal weight is studied experimentally for out-of-plane movement of a flat plate. The resolution becomes better when the number of windows becomes larger. However, the spatial resolution may be lower when the number of windows becomes larger. Therefore, the effects of the number of windows, the window size, the displacement resolution and the spatial resolution on the accuracy are also studied.

Holography has basically three-dimensional information. It is useful to analyze threedimensional displacement and strain distributions of an object. (Zhang et al., 1998; Kolenovic et. al., 2003) In this study, the Windowed PSDHI is extended to analyze threedimensional displacement components. The authors developed three systems for digital holographic interferometry using three-directional illuminations. The first one was for a laboratory bench system using a microscope (Morimoto et al., 2008a). The second one used collimated light beams from one laser source and 3 shutters (Fujigaki et al., 2005). It provided a stable system for static measurement (Shiotani et al., 2008). The third one used three spherical waves from three laser sources. It provided a compact system for static and dynamic measurement. In this study, after discussing the relationship between phase differences and displacement components, examples of three measurements using microscope, collimated light beams, and three spherical waves from three laser sources, are introduced.

\section{Principle of phase-shifting digital holographic interferometry}

\subsection{Principle of phase-shifting digital holography (Yamaguchi et al., 1997; Zhang et al., 1998)}

As an example of phase-shifting digital holography, a Twyman-Green type interferometer shown in Figure 1 is used. A collimated light from a laser is divided into an object wave and a reference wave by a beam splitter. The phase of the reference wave is shifted by $\alpha$ with a PZT stage. The value $\alpha$ is set as $0, \pi / 2, \pi$ and $3 \pi / 2$ in this phase-shifting digital holography. The four phase-shifted digital holograms are recorded on the CCD plane in a CCD camera without any focusing lens. The intensity of the hologram with a phase-shift value $\alpha$ at the pixel coordinates $(X, Y)$ on the CCD plane is expressed as $I(X, Y, \alpha)$. The amplitude $a_{0}(X, Y)$, the phase $\Phi_{0}(X, Y)$ and the complex amplitude $g(X, Y)$ of the object wave are expressed at the pixel coordinates $(X, Y)$ on the CCD plane as follows, respectively. 


$$
\begin{gathered}
a_{o}(X, Y)=\frac{1}{4} \sqrt{\left\{I\left(X, Y, \frac{3 \pi}{2}\right)-I\left(X, Y, \frac{\pi}{2}\right)\right\}^{2}+\{I(X, Y, 0)-I(X, Y, \pi)\}^{2}} \\
\tan \Phi_{o}(X, Y)=\frac{I\left(X, Y, \frac{3 \pi}{2}\right)-I\left(X, Y, \frac{\pi}{2}\right)}{I(X, Y, 0)-I(X, Y, \pi)} \\
g(X, Y)=a_{o}(X, Y) \exp \left\{i \Phi_{o}(X, Y)\right\}
\end{gathered}
$$

By calculating the Fresnel diffraction integral from the complex amplitudes $g(X, Y)$ on the CCD plane, the complex amplitude $u(x, y)$ of the reconstructed image at the position $(x, y)$ on the reconstructed object surface being at the distance $R$ from the CCD plane is expressed as follows.

$$
u(x, y)=\exp \left[\frac{i k\left(x^{2}+y^{2}\right)}{2 R}\right] F\left\{\exp \left[\frac{i k\left(X^{2}+Y^{2}\right)}{2 R}\right] g(X, Y)\right\}
$$

where $k$ and $F$ denote the wave number of the light and the operator of Fourier transform, respectively. The optical axis is normal to the CCD plane and the origin is at the center of the CCD plane. By calculating the intensities of these complex amplitudes on the reconstructed object surface, a holographically reconstructed image is obtained.

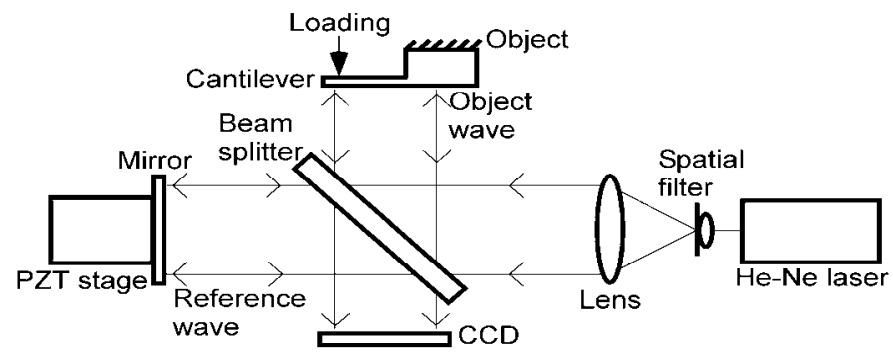

Fig. 1. Twyman-Green type interferometer for phase-shifting digital holography

\subsection{Principle of holographic interferometry}

Let us consider the deformation of an object (cantilever) shown in Figure 1. If the out-ofplane displacement $w(x, y)$ of the object is small, the amplitudes of the reconstructed object before and after deformation are almost the same and only the phase changes by $\Delta \phi(x, y)$ i.e. the phase-difference before and after the deformation. The relationship between the out-ofplane displacement $w(x, y)$ and the phase-difference $\Delta \phi(x, y)$ is expressed as follows.

$$
w(x, y)=\frac{\lambda}{4 \pi} \Delta \phi(x, y)
$$

where $\lambda$ is the wavelength of the light source.

\subsection{Experiment of displacement measurement (Morimoto et al., 2007)}

As an experiment, the deflection of a cantilever is measured using the optical system shown in Figure 1. The light source is a He-Ne laser. The power is $8 \mathrm{~mW}$ and the wavelength $\lambda$ is 632.8 
$\mathrm{nm}$. The pixel size of the CCD sensor is $4.65 \mu \mathrm{m}$ by $4.65 \mu \mathrm{m}$. A captured image is sampled by $1280(\mathrm{~V})$ by $960(\mathrm{H})$ pixels and the image is digitized with 8 bits. The image of $960(\mathrm{~V})$ by $960(\mathrm{H})$ pixels near the center of the recorded image is used for the analysis in this study.

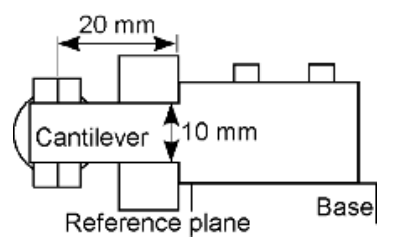

(a) Front view

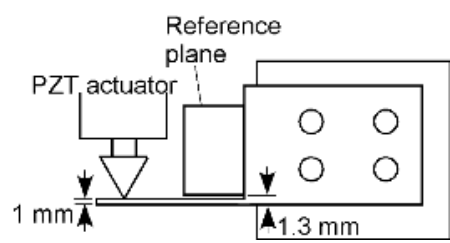

(b) Top view

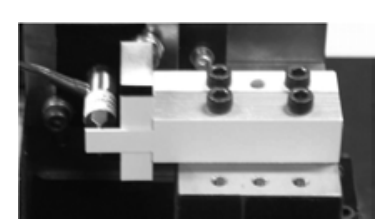

(c) Photograph

Fig. 2. Specimen (Cantilever)

As a specimen, a cantilever shown in Figure 2 is analyzed. (In this experiment, there is not a reference plane shown in the figure. The reference plane is used in Section 4). The cantilever is cut out from a thick stainless steel plate. The cantilever size is $10 \mathrm{~mm}$ wide, $25 \mathrm{~mm}$ long and $1 \mathrm{~mm}$ thick. The loading point is $20 \mathrm{~mm}$ from the fixed end. The displacement at the loading point is given by a micrometer with a wedge. To improve the reflection from the specimen, lusterless white lacquer is sprayed on the surface of the cantilever. The distance $\mathrm{R}$ from the CCD to the cantilever is $280 \mathrm{~mm}$. The phase of the reference wave is shifted by every $\pi / 2$ using a mirror controlled with a PZT stage. Then four phase-shifted digital holograms for one cycle of the phase-shifting are recorded in the memory of a personal computer. The complex amplitude of the brightness at each pixel on the hologram is calculated using the phase-shifting method expressed in Equations (1) to (3). The reconstructed complex amplitude of the object wave at a point of the reconstructed object surface is calculated from the complex amplitudes of the holograms using the Fresnel diffraction integral expressed in Eq. (4).

After the cantilever is deformed, the reconstructed complex amplitude of the object waves at the same point of the reconstructed object is obtained similarly. The reconstructed images and the phase distributions are obtained from the holograms with $960 \times 960$ pixels before and after deformation. The phase-difference distribution before and after deformation, that is, the out-of-plane displacement distribution is shown in Figure 3(a).

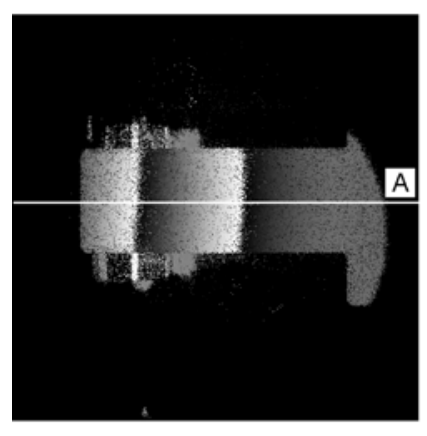

(a) From whole hologram $(n=1)$

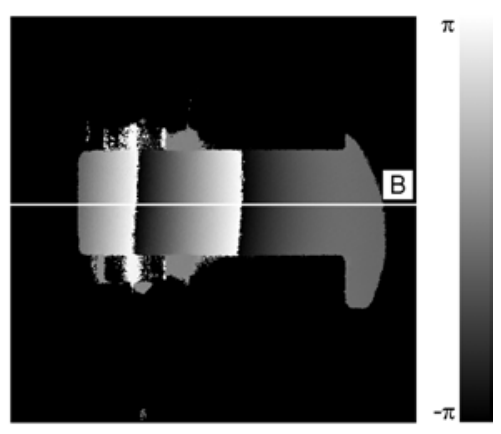

(b) From divided holograms $(n=16)$

Fig. 3. Phase-difference distributions obtained by digital holographic interferometry 


\section{Reduction method of speckle noise error by averaging phase-differences obtained by different windowed holograms (Morimoto et al., 2007)}

\subsection{Windowed hologram}

As mentioned above, the phase-difference is obtained from the complex amplitudes of the reconstructed holographic object before and after deformation. However, because coherent light is reflected from a rough surface, it provides random speckle patterns in the reconstructed image. And also, any measurement system has measurement error basically. Therefore, the obtained displacement distribution has also noise and the results are not so accurate. At the point where the intensity of the speckle is weak, the accuracy of the phase value of the light at the point becomes low. In holography, any part of a hologram has the optical information of the whole reconstructed image. By using this feature of holograms, the hologram is divided into many parts. The phase-difference at the same reconstructed point obtained from any part of the hologram should be the same if there is no error. The phase-difference obtained from the complex amplitude with high intensity is more reliable than the phase-difference obtained from the complex amplitude with low intensity. If there is speckle noise, among the phase-differences obtained from each divided hologram, the phase-difference with higher intensity at a reconstructed point is more reliable. Therefore, in our previous papers, the phase-difference was obtained by selecting the phase-difference with the maximum intensity at the same point (Morimoto et al. 2004, 2005a), or the phasedifference at the same point was obtained by averaging the phase-differences obtained from all the divided holograms by considering the weight of the intensity (Morimoto et al. 2005b, 2005c, 2007). It provided the displacement distribution with high-resolution.

In this section, the divided holograms are considered as windowed holograms using some window functions. A window function with value 1 in a small part of the whole hologram area and value 0 in the remaining area is superposed on an original hologram. By multiplying the window function with values 1 and 0 by the complex amplitude of the original hologram, the windowed hologram is obtained. By changing the position of the area with value 1 in the window function, many windowed holograms are formed. The reconstructed object image is calculated from each windowed hologram using Eq. (4). A point of the reconstructed object image has a speckle pattern. The speckle patterns obtained by different windowed holograms are all different from each other. However, the speckle pattern does not move as a result of small deformation but the phase is changed by the deformation. The intensity distributions of the reconstructed object and the phase-difference distribution before and after deformation are obtained from the windowed holograms with the same window function before and after deformation. After calculating the average intensity before and after deformation from $n$ sets of the windowed holograms obtained from the different window functions, the average value of the $n$ phase-difference values weighted proportional to the average intensity before and after deformation is calculated at each reconstructed point. The resultant average phase-difference value is highly reliable.

In this study, especially, the effect of the size or the number $n$ of the windows is examined. At first, for an example, let us consider the case of $n=16$. The hologram is divided into 16 square areas. That is, a window with value 1 in a square of $240 \times 240$ pixels and value 0 in the other area in the $960 \times 960$ pixels is superposed on the original hologram with $960 \times 960$ pixels. By moving the area with value 1 in the window, 16 windowed holograms are obtained and they are numbered as shown in Figure 4(a). The reconstructed object image is calculated from each windowed hologram using Eq. (4). The 16 intensity distributions of the 
reconstructed object images before deformation are shown in Figure 4(b). At each point on the reconstructed object, 16 complex amplitudes are obtained. Sixteen phase-difference values before and after deformation at each point are obtained. The average phasedifference is calculated by considering the weight of the average intensity before and after deformation. The result is shown in Figure 3(b) though the details of the displacement are described later. It provides more accurate phase-difference distribution than that shown in Figure 3(a).

In order to check the effect of the number of the windows on accuracy, the hologram is divided into $n$ square areas by changing $n(n=1,4,16,64,256$ and 1024). That is, the original hologram with $960 \times 960$ pixels is windowed with $n$ window functions whose small square area has value 1 and the remaining area has value 0 in the each window as in Figure 4(a). The average values of the $n$ phase-difference values obtained from the windowed holograms are calculated. The results are shown later.
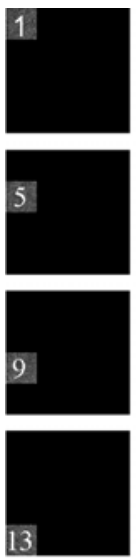

(a) Divided holograms
14
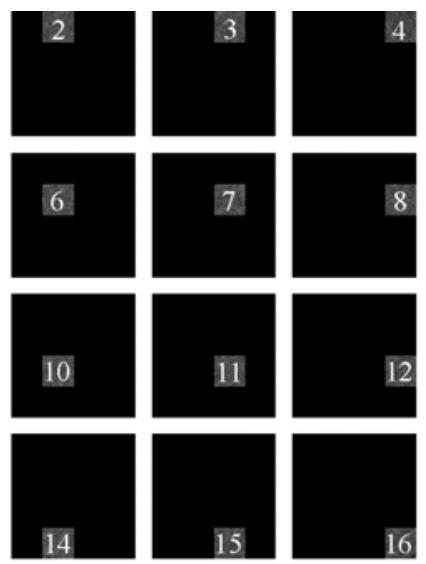

16

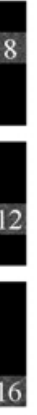

西

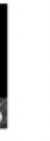

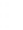

Fig. 4. Divided holograms and reconstructed object images

\subsection{Effect of number of windowed holograms on accuracy}

The effect on accuracy of changing the number of windowed holograms is examined. By changing the number $n$ into 1, 4, 16, 64, 256 and 1024, the displacement distributions are obtained. The displacement distributions along the centerline of the beam, shown as lines $\mathrm{A}$ and B in Figure 3 are shown in Figures 5(a) to 5(f). The theoretical displacement distribution for a cantilever is obtained by fitting a cubic curve with the minimum error by the least square method to each obtained distribution from the fixed point to the loading point. The standard deviations of the errors from the theoretical cubic curves are shown in Table 1 and Figure 6. The standard deviation decreases according to the number $n$, and the value becomes $670 \mathrm{pm}$ when $n=1024$. It shows the proposed method provides very high-resolution measurement. 


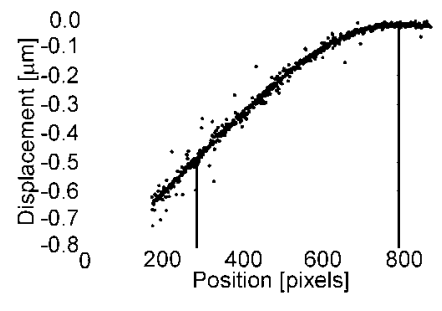

(a) $n=1$
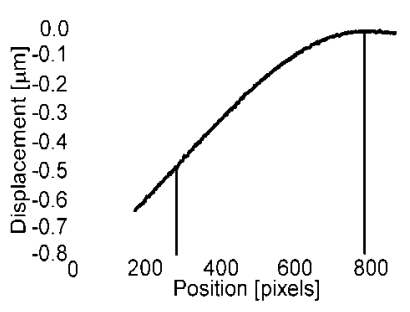

(c) $n=16$

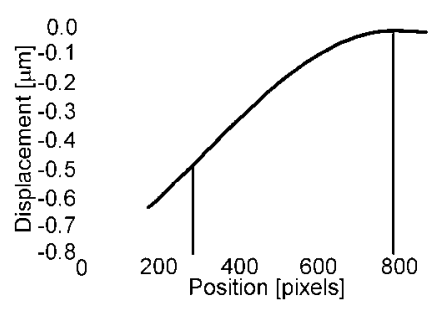

(f) $n=1024$

Fig. 5. Displacement distributions along lines A and B shown in Figure3

\subsection{Effect of weight of averaging on accuracy}

The effect on accuracy of changing the number of windowed holograms and the weight of the averaging is examined. When the weight is proportional to the $m$-th power of the amplitude of the complex amplitude, the standard deviations of errors are compared by changing the number $m$. In Section 3.2, the case of $m=2$ as the weight was examined by considering that reliability is larger according to the intensity, i.e., the 2nd power of the absolute amplitude of the complex amplitude. In this section, though the theoretical curve of the cantilever mentioned in the previous chapter is a cubic function, the experimental curve is not cubic in a precise sense because of the anisotropy of the materials, the actual boundary conditions, etc. In this section, parallel movement of a flat plate is adopted because the anisotropy of the materials, the actual boundary conditions, etc. are almost cut off. The parallel movement is a half wavelength, that is, about 316 nano- meters along the direction of the normal to the flat surface. The displacement distributions are shown in Figure 7 when $n=1$ and 1024 and $m=2$.

By changing 1, 4, 16, 64, 256 and 1024 as the number $n$, and 1/4,1/2, 1, 2, 4 and 8 as $m$ for the $m$-th power of the amplitude of the complex amplitude for the weight of the averaging of the phase-difference, i.e., the displacement distributions, the errors are examined. The displacement distributions along the centerline of the flat plate are analyzed by phaseshifting digital holographic interferometry. The theoretical displacement distribution for the centerline is obtained by fitting a linear expression with the minimum error by the least square method to each obtained distribution. The standard deviations of the errors from the theoretical linear expressions are shown in Figure 8. The standard deviation decreases according to the number $n$, as same as in the case of Section 3.2. By changing $n$ and $m$, the standard deviation is examined. In the same $n$, the standard deviation is the minimum when $m$ is 2 , that is, when the weight is proportional to the intensity of the complex amplitude. It is appropriate to adopt $m=2$ as the weight of averaging. The minimum standard deviation of errors is 88 pico-meters when $n=1024$ and $m=2$.

\begin{tabular}{c|c|c|c|c|c|c}
\hline \hline Number of windows $n$ & 1 & 4 & 16 & 64 & 256 & 1024 \\
\hline Standard deviation [nm] & 16.39 & 4.02 & 1.95 & 1.09 & 0.78 & 0.67
\end{tabular}

Table 1. Relationship between number of windows and standard deviation of errors 


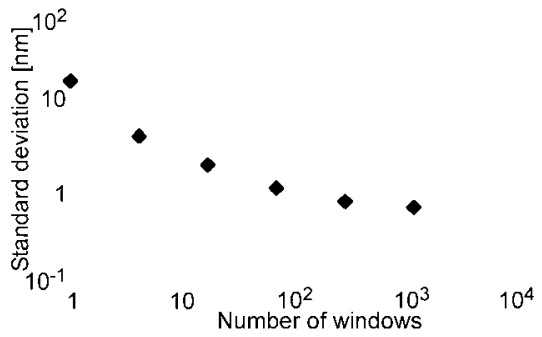

Fig. 6. Relationship between number of windows and standard deviation of errors

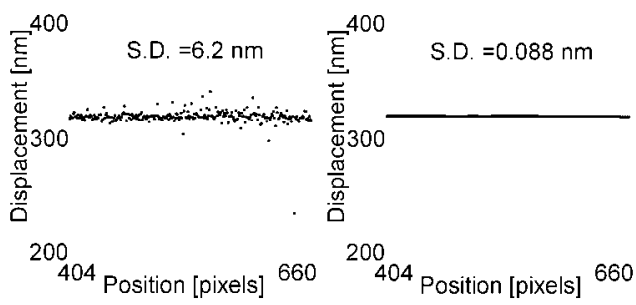

$\begin{array}{ll}\text { (a) } n=1 & \text { (b) } n=1024\end{array}$

Fig. 7. Displacement distributions when flat plate is moved out of plane

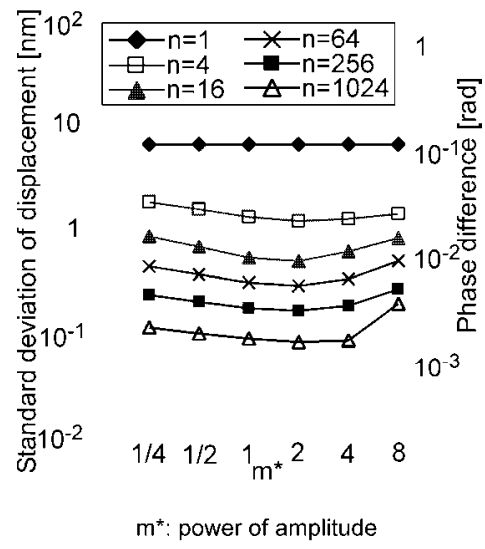

Fig. 8. Standard deviations of errors when number of windows and power of the amplitude as weight of averaging are changed

\section{Effect of window size on accuracy and spatial resolution in windowed phase-shifting digital holographic interferometry (Morimoto, 2008b)}

As shown in Figure 6, the accuracy becomes better according to the number of windows. However, the spatial resolution may be lower when the number of windows becomes larger. In this section, therefore, the effects of the number of windows, the window size, the displacement resolution and the spatial resolution on the accuracy are analyzed. 


\subsection{Displacement measurement of cantilever by phase-shifting digital holographic interferometry}

As an experiment, the deflection of a cantilever is also measured using the optical system shown in Figure 1. As a specimen, a same size cantilever shown in Figure 2 is also analyzed. In this experiment, a fixed reference plate is set at $1.3 \mathrm{~mm}$ behind the cantilever to check spatial resolution, as shown in Figure 1.

The reconstructed images (the intensity distribution, i.e., the amplitude squared) and the phase distributions are obtained from the holograms with $960 \times 960$ pixels before and after deformation. The reconstructed image before deformation is shown in Figure 9(a). The phase-difference distribution before and after deformation, that is, the out-of-plane displacement distribution is shown in Figure 10(a).

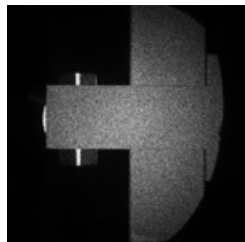

(a) $n=1$

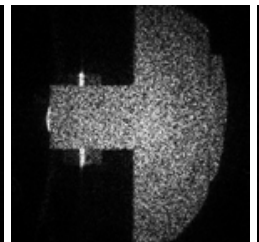

(b) $n=16$

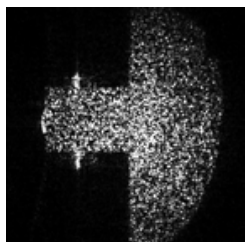

(c) $n=64$

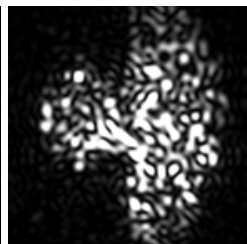

(d) $n=1024$

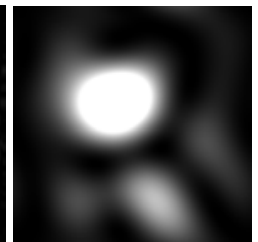

(e) $n=57600$

Fig. 9. Reconstructed image obtained by Windowed PSDH

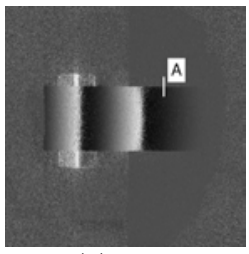

(a) $n=1$

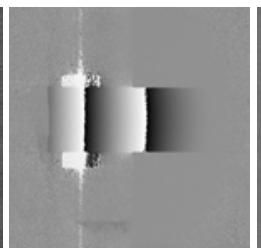

(b) $n=16$

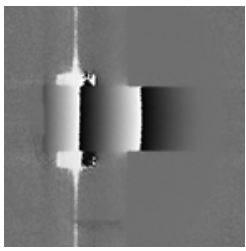

(c) $n=64$

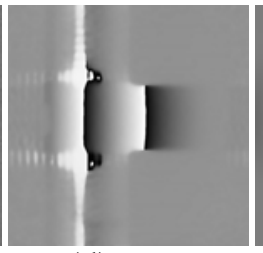

(d) $n=1024$

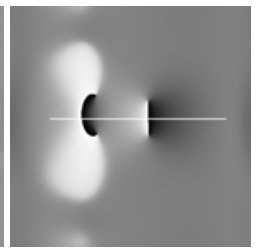

(e) $n=57600$

Fig. 10. Phase difference distribution obtained by Windowed PSDH

\subsection{Effect of number of windowed holograms on accuracy and computation processing time}

The effect on accuracy by changing the number $n$ of windowed holograms into 1, 4, 16, 64, 256, 1024, 4096, 14400, 25600 and 57600 is examined. Some reconstructed images obtained from each one of the windowed holograms are shown in Figure 9. When the number of windows becomes larger, that is, the window size becomes smaller, the speckle size becomes larger. It is considered that the spatial resolution would be worse when the speckle size becomes larger. Figure 10 shows some of the results. The displacement distributions along the centerline of the beam are shown in Figure 11. The theoretical displacement distribution for a cantilever is obtained by fitting a cubic curve with the minimum error by means of the least square method to each obtained distribution from the fixed point to the loading point. The standard deviations of the errors from the theoretical cubic curves are shown in Table 2 and Figure 11. The standard deviation changes according to the number $n$. Though the standard deviation when $n=1$ is $22 \mathrm{~nm}$, it is $2.4 \mathrm{~nm}$ when $n=16$. The value becomes the minimum value of $680 \mathrm{pm}$ when $n=1024$. It shows the proposed method 
provides very high-resolution measurement by selecting appropriate number $n$. The calculation time is, however, almost proportional to the number $n$ as shown in Table 2 and Figure 13. In practice, 16, 64 or 256 windowed holograms are recommended by considering the balance of the computation time and accuracy.

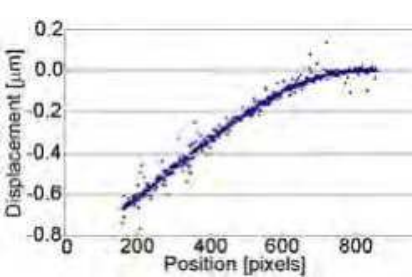

(a) $n=1$

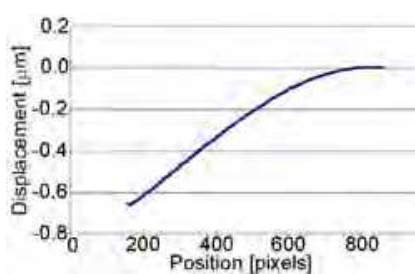

(c) $n=64$

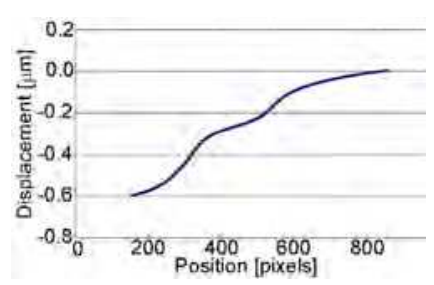

(e) $n=57600$

Fig. 11. Displacement distributions along centerline of cantilever of Figure 4

\begin{tabular}{|c|c|c|c|c|c|}
\hline \multirow{2}{*}{$\begin{array}{l}\text { Number of } \\
\text { window } n\end{array}$} & \multirow{2}{*}{$\begin{array}{l}\text { Window } \\
\text { size } \\
\text { [pixels] }\end{array}$} & \multicolumn{2}{|c|}{ Standard deviation } & \multirow{2}{*}{$\begin{array}{l}\text { Processing } \\
\text { time[s] }\end{array}$} & \multirow{2}{*}{$\begin{array}{l}\text { Spatial } \\
\text { resolution } \\
\text { [pixel] }\end{array}$} \\
\hline & & {$[\mathrm{nm}]$} & [rad] & & \\
\hline 1 & 960 & 21.93 & 0.435 & 3 & 0.36 \\
\hline 4 & 480 & 4.87 & 0.097 & 9 & 0.44 \\
\hline 16 & 240 & 2.35 & 0.047 & 29 & 1.5 \\
\hline 64 & 120 & 1.61 & 0.032 & 110 & 2.8 \\
\hline 256 & 60 & 0.89 & 0.018 & 430 & 6.66 \\
\hline 1024 & 30 & 0.68 & 0.013 & 1704 & 16.24 \\
\hline 4096 & 15 & 0.94 & 0.019 & 6791 & 27.68 \\
\hline 14400 & 8 & 2.29 & 0.045 & 23751 & - \\
\hline 25600 & 6 & 2.8 & 0.056 & 42237 & - \\
\hline 57600 & 4 & 15.7 & 0.312 & 95430 & - \\
\hline $\begin{array}{c}\text { Smoothing } \\
11 \times 11\end{array}$ & -- & 1.69 & 0.034 & 4 & 6.77 \\
\hline
\end{tabular}

Table 2. Relationship among number of windows, standard deviation of error and processing time

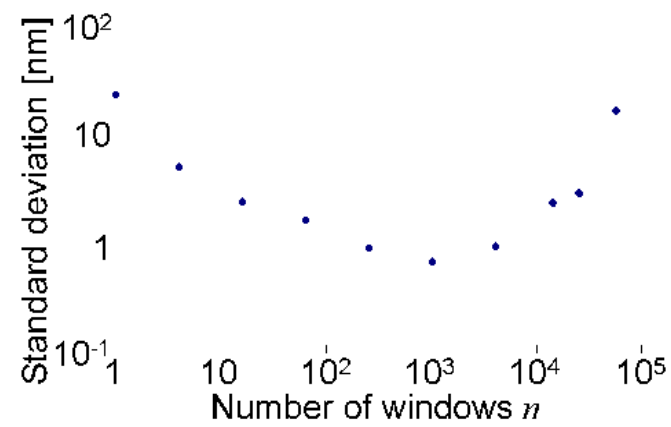

Fig. 12. Relationship between number of windows and standard deviation 


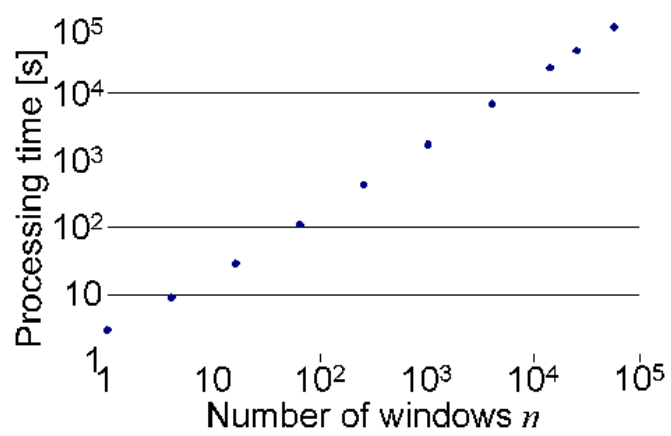

Fig. 13. Relationship between number of windows and processing time

\subsection{Effect of window size on standard deviation of errors and spatial resolution}

As shown in Figures 9(e) and 10(e), large speckles cannot provide accurate results. In this section, the relationship between window size and spatial resolution is examined. The phase-difference distributions along the vertical white line A $(x=632$ pixels, $y=260-380$ pixels) in Figure 10(a) are shown in Figure 14. The theoretical phase difference along the line A on the cantilever is $\Pi / 2$. The theoretical phase difference along the line A on the fixed back plate is 0 . Although the theoretical phase-difference distribution has a discontinuity at the edge of the cantilever $(y=318)$, the analysed phase-difference distributions are continuous slopes. Figure 14 also shows the result obtained by a conventional averaging method using values at the peripheral $11 \times 11$ pixels. However, the spatial resolution obtained from a small window may be bad. That is, bad spatial resolution changes a step function along line A into a continuous function. In order to define the spatial resolution quantitatively in this study, it is defined as the distance (in pixels) between the positions at the $25 \%$ and $75 \%$ values of the step of the phase difference as shown in Figure 15.

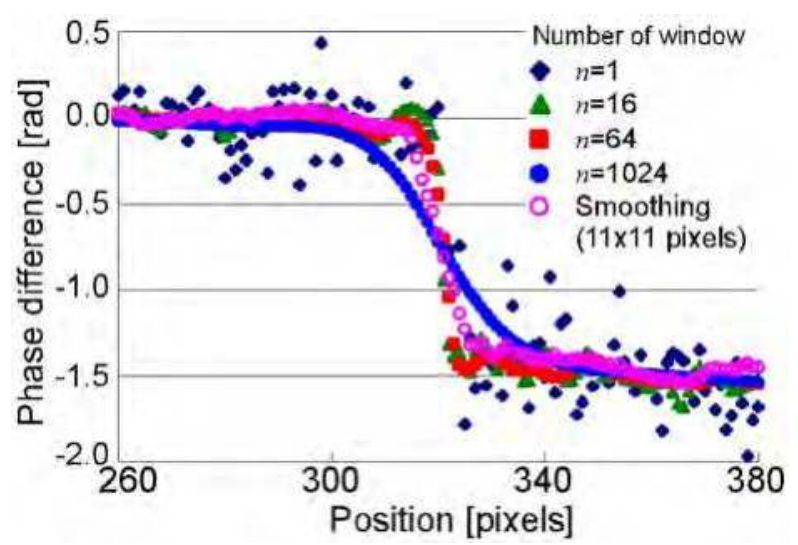

Fig. 14. Phase difference distribution along line A in Figure 10

The results of the relationships between the number of windows, the standard deviation of error and the spatial resolution are shown in Table 2 and Figure 16. When the window size becomes 
smaller, the standard deviation of errors becomes better and the spatial resolution becomes worse. The computation time becomes worse. By comparing the spatial resolution (6.77 pixels) of the conventional averaging method using $11 \times 11$ pixels and the spatial resolution $(2.80$ pixels) when the number of windows is 64, which have almost the same standard deviation of error, the windowed PSDHI is better than the conventional averaging method.

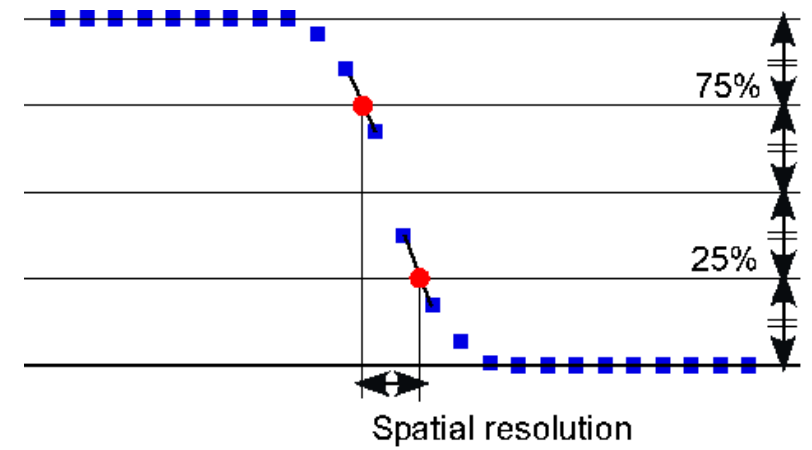

Fig. 15. Definition of special resolution

Although the standard deviation of error when $n=1024$ is the best in Table 2, the data when $n=1024$ in Figure 14 show a bad slope function far from the step function. Although the result from the hologram with a small window has good accuracy in an area with almost constant values of intensity near the centerline, it is not very accurate in the area with nonconstant values near the edge because of large speckle size.

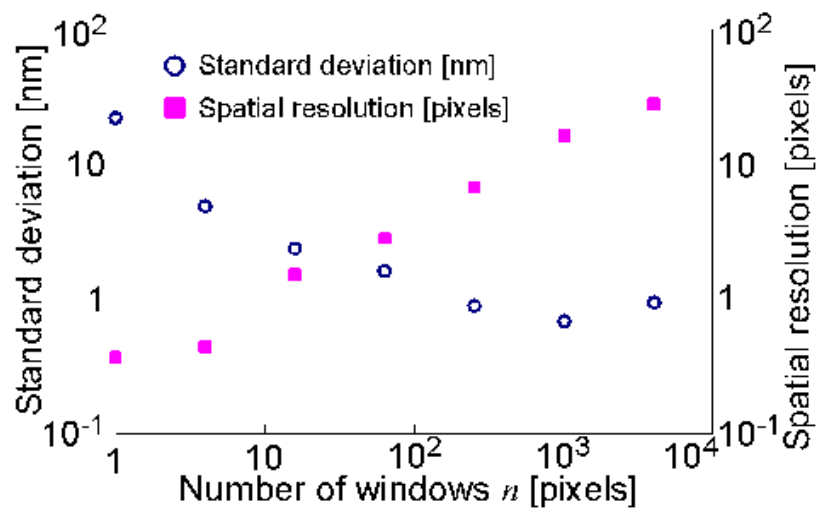

Fig. 16. Standard deviation and spatial resolution against number of windows in Table 2

\subsection{Effect of larger window size on standard deviation of error and spatial resolution} In the previous section, the effect of window size on the standard deviation of error and the spatial resolution were examined. However, the numbers of windows are different. In this section, the effect of window size on the standard deviation of error and the spatial resolution are examined when the numbers of the windows are the same $(n=256)$. The standard deviation and the spatial resolution when the window sizes are 480, 240, 120, 60 
and 30 are shown in Table 3 and Figure 17. The spatial resolution becomes worse when the window size becomes smaller. The standard deviation becomes also better when the window size becomes smaller. However, the standard deviation is the best when the window size is 60 . The standard deviation when the window size is 30 is worse than that when the window size is 60 . Although the case $w=60$ and $n=256$ uses all the data of the hologram, the case $w=30$ and $n=256$ does not use all the data of the hologram. Large speckles cannot provide accurate results.

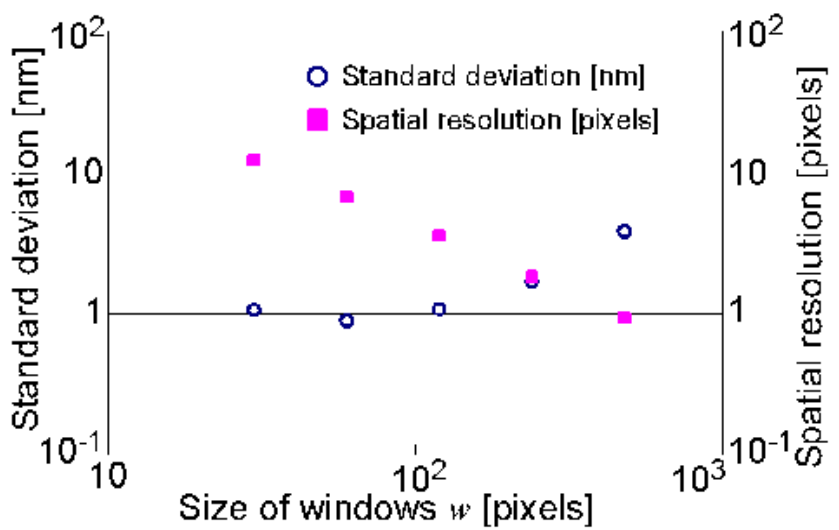

Fig. 17. Relationship between standard deviation and spatial resolution in Table 3

\begin{tabular}{ccccc}
\hline \hline $\begin{array}{c}\text { Window } \\
\text { size } w\end{array}$ & $\begin{array}{c}\text { Number of } \\
\text { windows } n\end{array}$ & $\begin{array}{c}\text { Standard } \\
\text { deviation [nm] }\end{array}$ & $\begin{array}{c}\text { Spatial resolution } \\
\text { [pixels] }\end{array}$ & $\begin{array}{c}\text { Processing time } \\
{[\mathrm{s}]}\end{array}$ \\
\hline 480 & 256 & 3.81 & 0.93 & 493 \\
240 & 256 & 1.68 & 1.81 & 457 \\
120 & 256 & 1.07 & 3.55 & 439 \\
60 & 256 & 0.89 & 6.66 & 430 \\
30 & 256 & 1.06 & 12.16 & 428 \\
\hline
\end{tabular}

Table 3. Relationship among Window size, standard deviation of error and spatial resolution

In this windowed PSDHI study, the effect of the number of windows or window size on accuracy, spatial resolution and computation time were examined. When the number of windows increases, accuracy becomes better at first because of speckle noise reduction and then it becomes worse because of larger speckle size. The best accuracy is of the subnanometer scale when the number of windows is 1024 in our experiment. However, the accuracy is better than that when using the conventional averaging method with $11 \times 11$ pixels. The spatial resolution becomes worse when the number of windows becomes larger, and correspondingly the window size becomes smaller. If the number of windows is constant, the spatial resolution is better when the window size becomes larger and the accuracy is better when all the data are used and the window size is smaller. The computation time increases according to the number of windows. In practice, it is useful when the number of 
windows is 16,64 or 256 , as in our experiment by considering the balance of the computation time and accuracy.

\section{Three-dimensional displacement analysis by digital holographic interferometry}

In order to measure three-dimensional displacement components, a three-directionalillumination method or three-directional-observation method is usually employed (Zhang et al., 1998; Kolenovic et al. 2003). However, in order to analyze strain distributions, the displacement is spatially differentiated which requires accurate measurement of displacement distributions. Windowed PSDHI is useful for measuring quantitatively nanometer displacement of objects (Morimoto et al., 2007; 2008b). The authors previously proposed a method of simultaneous measurement of in-plane and out-of-plane displacements using two beam illuminations. (Okazawa et al. 2005) A three-directional illumination method was also proposed (Fujigaki et al. 2005; Morimoto et al. 2008a, 2008c). To miniaturize the equipment for practical use, laser beams with spherical wavefronts were used. However, if spherical waves are used, the incident angles are different for each point and then each point has different sensitivity vectors. The incident angle for each point on an object is determined by the three-dimensional position of the point and the point source of the laser beam. It is, however, difficult to measure the incident angle accurately. The authors proposed a calibration method with a reference flat plane. The reference plane was installed on an XYZ three-axis piezo stage which was movable in the XYZ directions by a very small amount (Morimoto et al., 2008a).

By calculating the each phase-difference between before and after deformation using digital holography, the parameter for the relationship between the displacement and the phasedifference can be obtained. Tabulation of parameters for each point helps to measure the displacement in high speed from the phase-difference of a specimen. Displacement measurement using spherical waves can be realized with this calibration method. The theoretical treatment and experimental results of some three-dimensional displacement measurements using this method are shown in this study.

\subsection{Relationship between phase-differences and displacement components}

The schematic positions of an object and an observation direction when the object is illuminated at a particular incident angle are shown in Figure 18. The phase-difference for a unit displacement depends on the position of the object (Morimoto et. al., 2008; Fujigaki et al., 2006). When the positional relationship is as expressed in Figure 18, the equation at a point $\mathrm{P}$ before deformation on an object is expressed as shown in Equation (6).

$$
\Delta \phi=e \cdot d
$$

where, $e$ is the sensitivity vector which depends on the half of the angle between the incident angle $\theta_{1}$ and the observation angle $\theta_{0}$ of the point $\mathrm{P}$, and $d$ is the displacement vector for the point $\mathrm{P}$ before deformation which moves to a point $\mathrm{P}^{\prime}$ after deformation. As the displacement is very small compared with the distance between the light source and the object, it is assumed that the incident angle $\theta_{1}$ and the observation angle $\theta_{0}$ do not change. And $\Delta \phi$ is the phase-difference resulted from the displacement at the point $P$.

The displacement vector $d$ and the sensitivity vector $e$ each have components in the $x, y$, and $z$ directions. Then Eq. 6 is written as 


$$
\Delta \phi=\left[\begin{array}{lll}
e_{x} & e_{y} & e_{z}
\end{array}\right]\left[\begin{array}{c}
d_{x} \\
d_{y} \\
d_{z}
\end{array}\right]=e_{x} d_{x}+e_{y} d_{y}+e_{z} d_{z}
$$

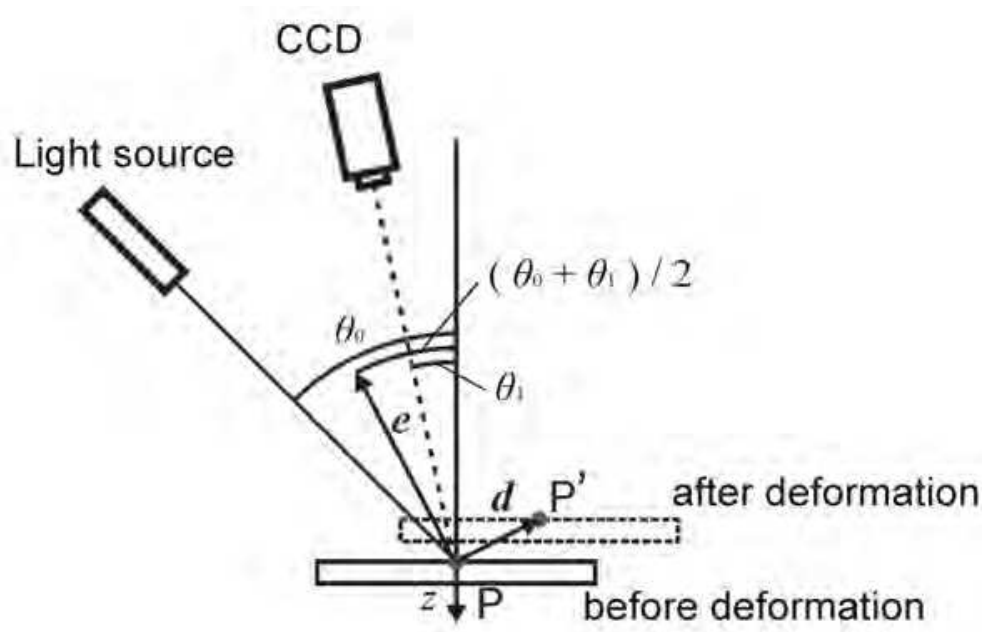

Fig. 18. Relationship between displacement of object, observation direction and incident angle at Point $\mathrm{P}$

When an object is illuminated from three different directions, the number of parameters of the sensitivity vector components increases, and Equation (7) can be extended as Equation (8);

$$
\begin{aligned}
{\left[\begin{array}{l}
\Delta \phi_{1} \\
\Delta \phi_{2} \\
\Delta \phi_{3}
\end{array}\right] } & =\left[\begin{array}{lll}
e_{1 x} & e_{1 y} & e_{1 z} \\
e_{2 x} & e_{2 y} & e_{2 z} \\
e_{3 x} & e_{3 y} & e_{3 z}
\end{array}\right]\left[\begin{array}{l}
d_{x} \\
d_{y} \\
d_{z}
\end{array}\right] \\
S & =\left[\begin{array}{lll}
e_{1 x} & e_{1 y} & e_{1 z} \\
e_{2 x} & e_{2 y} & e_{2 z} \\
e_{3 x} & e_{3 y} & e_{3 z}
\end{array}\right]
\end{aligned}
$$

where the suffixes 1, 2 and 3 show the corresponding illumination directions.

Each component of the sensitivity vector matrix $S$ is obtained by the geometric parameters of the optical system. When each component of the matrix $S$ of Equation (7) is specified, the displacement components $d_{x} d_{y}$ and $d_{z}$ can be obtained from the phase-difference $\Delta \phi_{1}, \Delta \phi_{2}$ and $\Delta \phi_{3}$ for each incident light, respectively, using the inverse matrix $S^{-1}$ of the sensitivity vector matrix $S$ as follows; 


$$
\begin{gathered}
{\left[\begin{array}{l}
d_{x} \\
d_{y} \\
d_{z}
\end{array}\right]=\left[\begin{array}{lll}
f_{x 1} & f_{x 2} & f_{x 3} \\
f_{y 1} & f_{y 2} & f_{y 3} \\
f_{z 1} & f_{z 2} & f_{z 3}
\end{array}\right]\left[\begin{array}{l}
\Delta \phi_{1} \\
\Delta \phi_{2} \\
\Delta \phi_{3}
\end{array}\right]} \\
S^{-1}=\left[\begin{array}{lll}
f_{x 1} & f_{x 2} & f_{x 3} \\
f_{y 1} & f_{y 2} & f_{y 3} \\
f_{z 1} & f_{z 2} & f_{z 3}
\end{array}\right]
\end{gathered}
$$

where $f_{i j}$ is the $(i, j)$ component of $S^{-1}$.

\subsection{Displacement and strain measurement of L-shaped cantilever}

\subsubsection{Laboratory system for microscope}

Figure 19 shows a schematic view of an optical system for digital holographic interferometry with a microscope. A parallel collimated laser beam illuminates an object. The light source is a He-Ne laser (Output: $8 \mathrm{~mW}$, Wavelength: $632.8 \mathrm{~mm}$ ). The laser beam from the light source is separated into three object beams ch.1, ch.2 and ch.3 and one reference beam by using three beam splitters, one half mirror and several mirrors. The reflected object beam arrives in a CCD plane through the microscope. A parallel reference beam is incident from a beam splitter between the object and the microscope. The reference beam also arrives in the same CCD plane. Each object beam is interfered with the reference beam by cutting off the other object beams. The phase of the reference beam is changed by using the PZT stage.

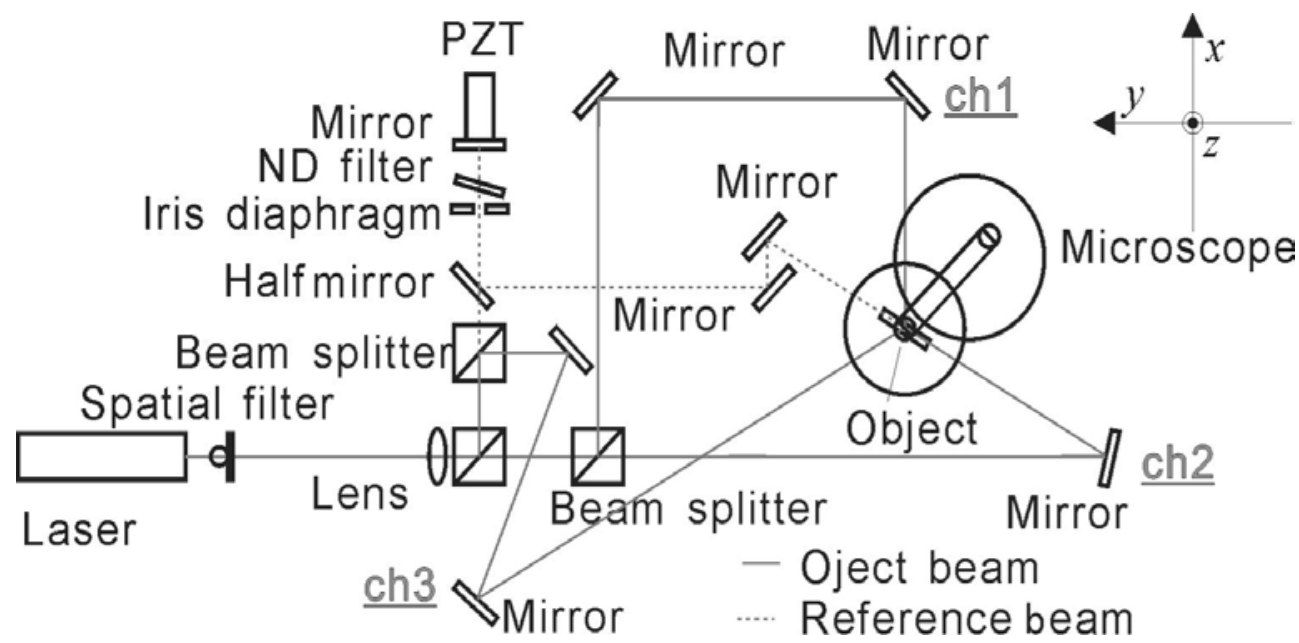

Fig. 19. Optical setup for experiment 
The pixel size of the used CCD (SONY XCD-900) is $4.65 \mu \mathrm{m} \times 4.65 \mu \mathrm{m}$. The analyzed image size is $960 \times 960$ pixels. A stereoscopic microscope (NIKON) using a CCD camera adapter is used. In the analysis, windowed PSDHI with 64 windows is used in order to decrease the effect of speckle noise.

Displacement measurement of an L-shaped cantilever is performed by using the experimental setup shown in Figure 19.

An L-shaped cantilever with a fixed end, $2 \mathrm{~mm}$ width, $8 \mathrm{~mm}$ length, and $1 \mathrm{~mm}$ thickness shown in Figure 20(a) is measured. Figure 20(b) shows the reconstructed image. Figures 20(c), (d) and (e) shows the phase-difference distribution respectively obtained by a laser beams ch1, ch2 and ch3 in Figure 19. Figures 20 (f) and (g) show the resultant threedimensional displacement component distributions along the lines $x_{0} x_{1}$ and $x_{1} x_{2}$ shown in Figs. 20 (c), (d) and (e).

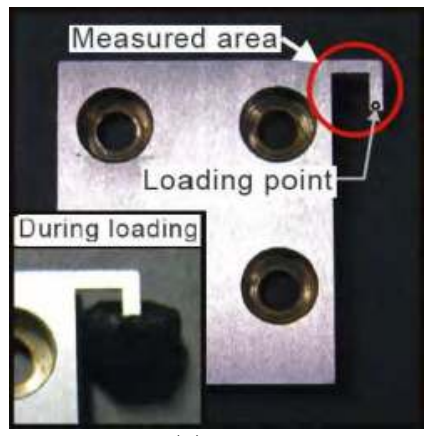

(a) Specimen

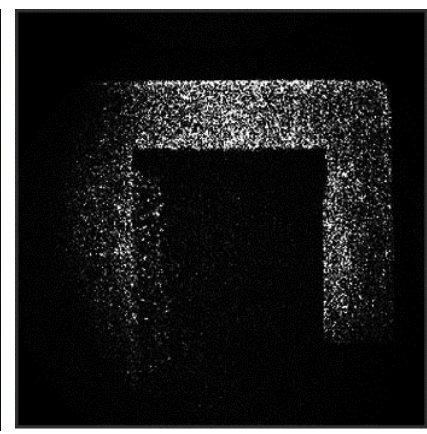

(b) reconstructed image

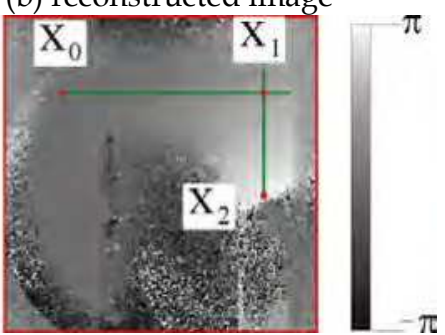

(e) $\Delta \phi_{3}$

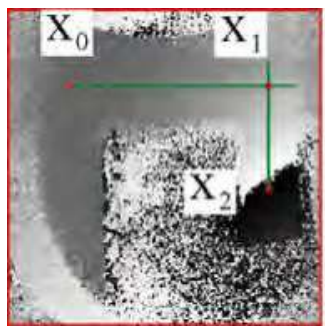

(c) $\Delta \phi_{1}$

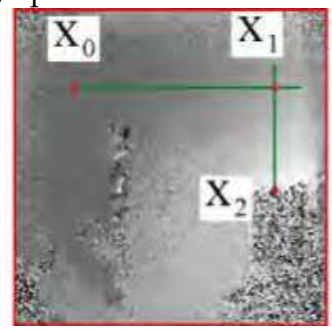

(d) $\Delta \phi_{2}$

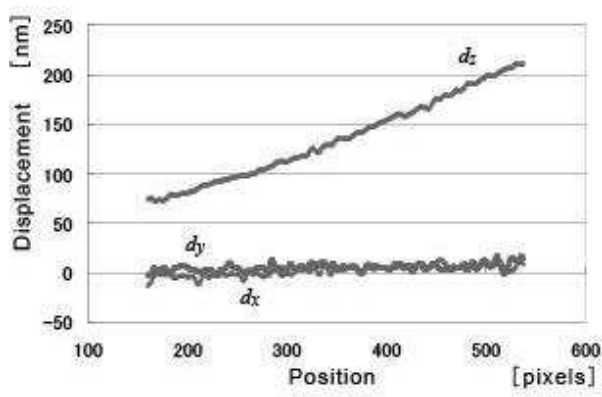

(g) Displacement components along $x_{1} x_{2}$

(f) Displacement components along $x_{0} x_{1}$

Fig. 20. Displacement measurement of L-shaped cantilever 


\subsubsection{Collimated wave system}

This system has two sets of two-beam illuminations. One set, that is, an optical setup for displacement measurement using two-beam illuminations is shown in Figure 21. A collimated light beam from a laser is divided into an object wave and a reference wave by beam splitter 1. Additionally, the object wave is divided into object wave 1 and object wave 2 by beam splitter 2 . Each object wave has a shutter on the light path. From the two holograms, the $x$-directional in-plane and the $z$-directional out-of-plane displacements are analyzed. Another set of two-beam illuminations provides the $y$-directional in-plane displacement.

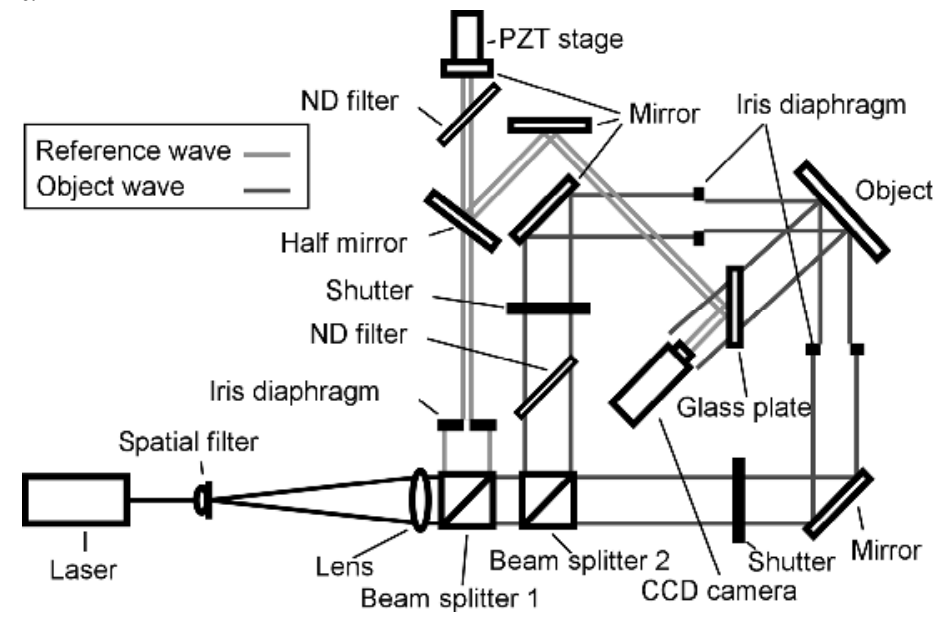

Fig. 21. Optical using two beam illuminations

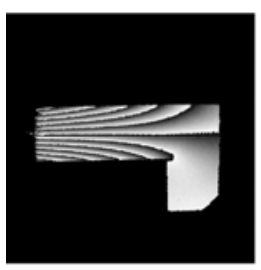

(a) $\phi_{x}$

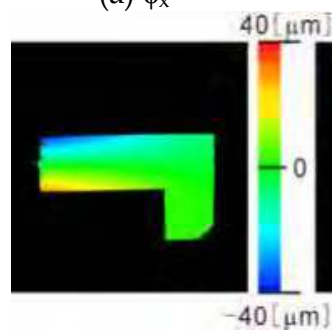

(e) $u_{\mathrm{x}}$

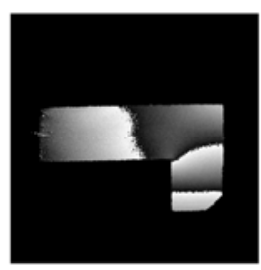

(b) $\phi_{\mathrm{z}}$

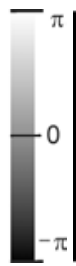

$100[\mu \mathrm{m}]$

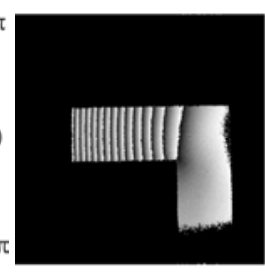

(c) $\phi_{\mathrm{y}}$

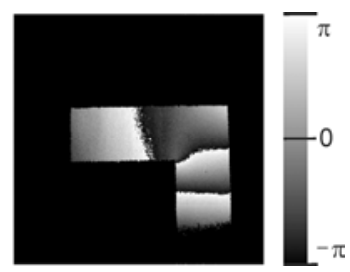

(d) $\phi_{\mathrm{z}}$

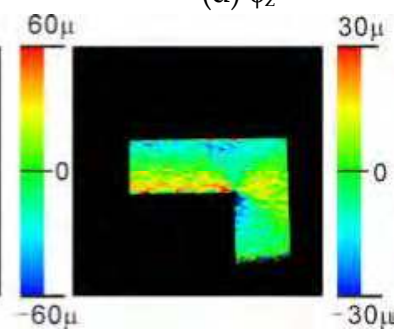

(h) $e_{y}$

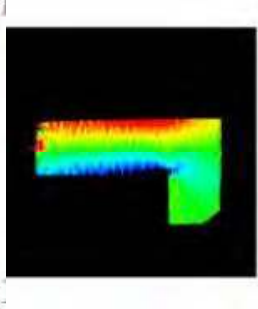

(g) $e_{x}$

(f) $u_{\mathrm{y}}$

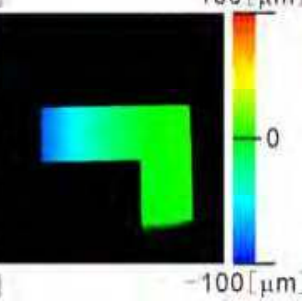

Fig. 22. Phase-difference displacement and strain distributions measured by collimated beam system 
An L-shaped cantilever with a fixed line is measured by the system shown in Figure 21. Figures 22(a) and (b) show the phase-difference distributions along the $x$ - and $z$-directions, respectively, obtained by the $x$-directional set. Figures 22(c) and (d) show the phasedifference distributions along the $y$ - and $z$-directions, respectively, obtained by the $y$ directional set. Figures 22(e) and (f) show the $x$ - and $y$-directional displacement distributions, respectively. Figures $22(\mathrm{~g})$ and $(\mathrm{h})$ show the $x$ - and $y$-directional strain distributions, respectively.

\subsubsection{Spherical wave system}

In this experiment, three holograms using light sources 1,2 and 3 are recorded on a CCD simultaneously as shown in Figure 23. Although the phases of the three reference waves are shifted simultaneously seven times, the phase-shift amounts during the seven times are different for each light source. The total phase-shifts are $2 \pi, 4 \pi$ and $6 \pi$ for the light sources 1 , 2 and 3 , respectively. The each fringe pattern by the light sources 1, 2 and 3 is extracted from the continuous seven holograms using the Fourier transformation of the brightness change at each pixel of the holograms.

Let us explain the procedures. Figure 24 illustrates the captured brightness changes at a pixel and the brightness change corresponding to each of the three light sources. The discrete Fourier transformation of the captured brightness along the time axis provides the Fourier spectrum shown in Figure 25. It has seven frequency components from -3 to 3 . Here, the components of the frequencies 1, 2 and 3 arise from the light sources 1, 2 and 3, respectively. By extracting these components and calculating the amplitudes and the phases of the components, the complex amplitudes of the three holographic fringe patterns are obtained separately.

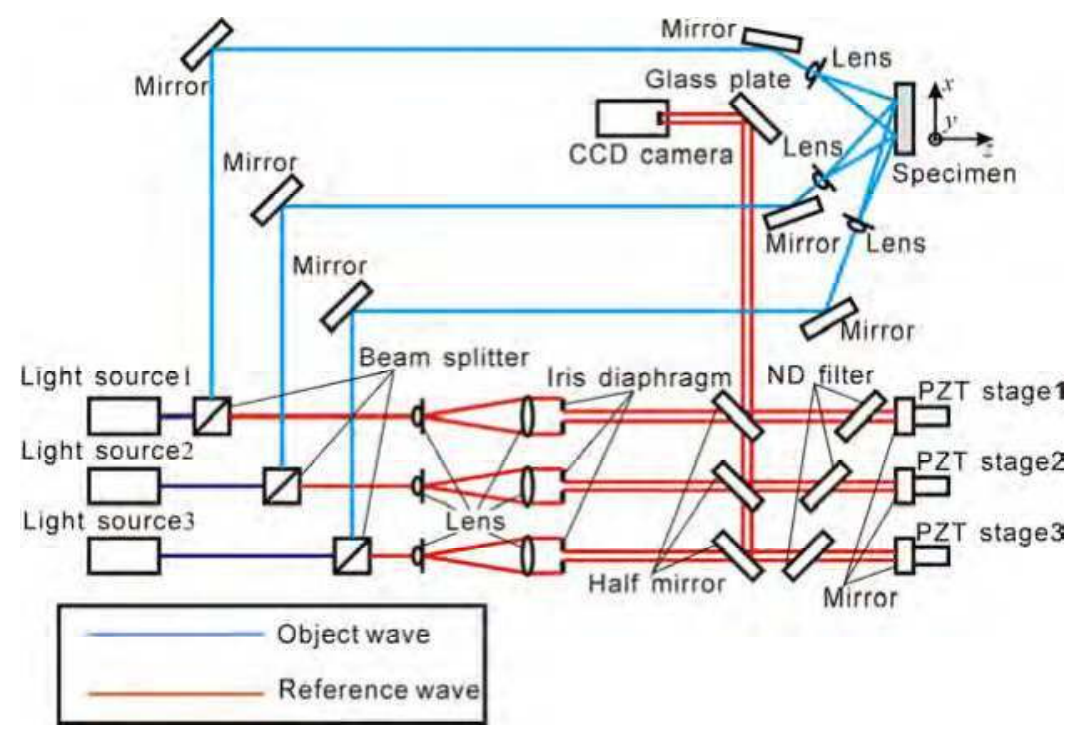

Fig. 23. Optical setup using three spherical waves 


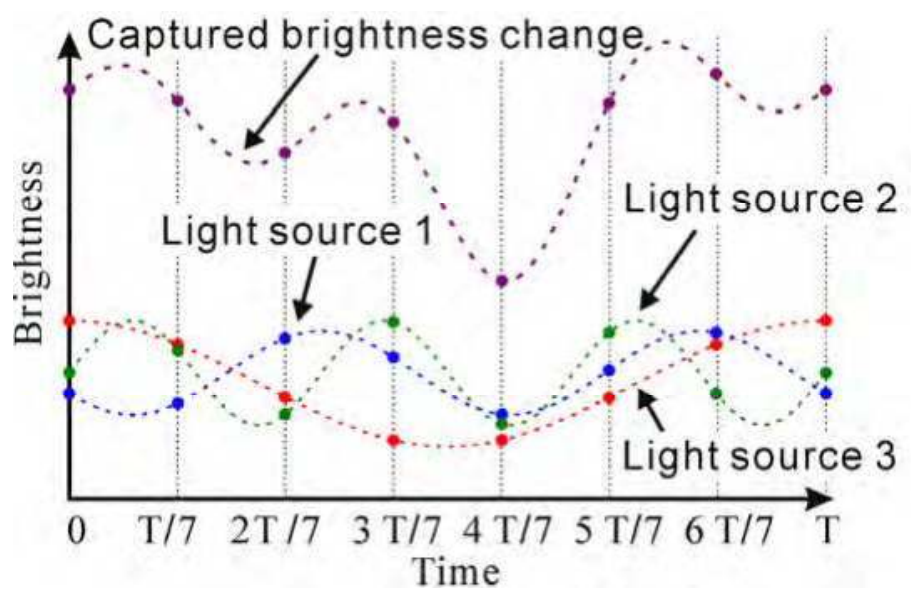

Fig. 24. Brightness change of composite wave

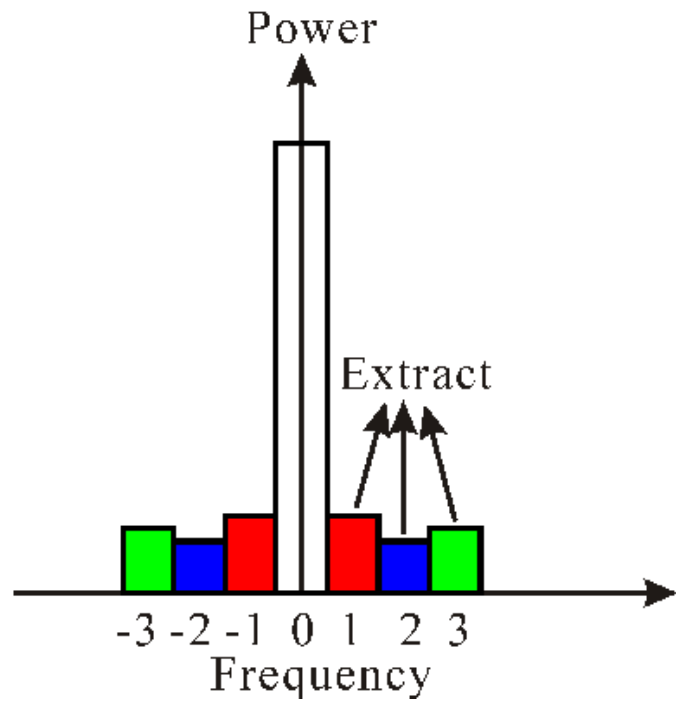

Fig. 25. Fourier spectrum of composite wave 
A strain distribution measurement of a T-shaped cantilever with a fixed line is performed by the system shown in Figure 23. The size of the cantilever is $5 \mathrm{~mm}$ width, $15 \mathrm{~mm}$ length, and $5 \mathrm{~mm}$ thickness. A load is applied on the cantilever at the point $12.5 \mathrm{~mm}$ from the base of the cantilever.

Seven phase-shifted holograms are captured before and after deformation. The Fourier components corresponding to each light source are separated by the phase-shifting method using Fourier transformation and each phase value is obtained. The phase-difference distributions of the light sources 1, 2 and 3 are shown in Figs. 28 (a), (b) and (c), respectively. The strain distribution in the $x$-direction is shown in Figure 27.

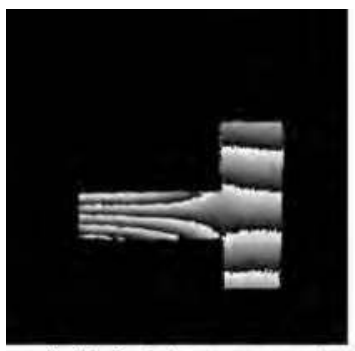

(a) Light source 1

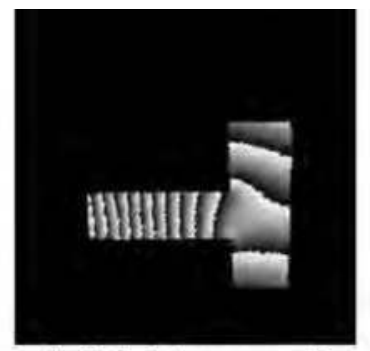

(b) Light source 2

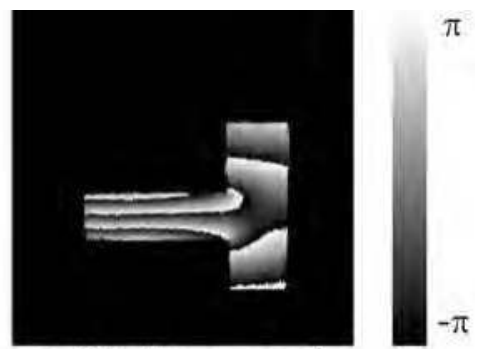

(c) Light source 3

Fig. 26. Phase-difference distributions by each light sources

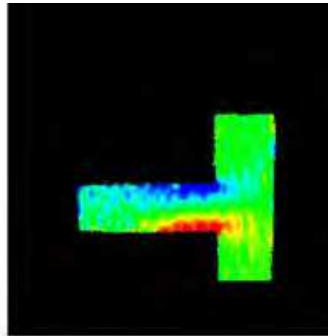

$\varepsilon_{s}$

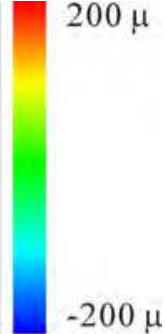

$-200 \mu$

Fig. 27. Strain distribution in $x$-direction

\section{Conclusions}

In order to reduce the effect of speckle noise, the windowed digital holographic interferometry was proposed. In the method, the effect of number of windows, or window size on accuracy, spatial resolution and calculation time were examined. The results are that the number of windows increases, the accuracy becomes better at first because of speckle noise reduction and after that it becomes worse because of larger speckle size. The best accuracy is sub-nanometer when the number of windows is 1024 in our experiment. 
However, the accuracy is better than the conventional averaging method with 11 by 11 pixels. The spatial resolution becomes worse when the number of windows becomes larger, that is, the window size becomes smaller. If the number of windows is constant, the accuracy and spatial resolution is better when the window size becomes larger. The calculation time increases according to the number of windows. In practice, it is useful when the number of windows is 16, 64 or 256 in our experiment. Three holographic systems were developed and applied to measure 3D displacement and strain distributions.

\section{Acknowledgment}

A part of this study was supported by a grant aided project for creation of new regional industry of Kansai Bureau of Economy Trade and Industry. The authors also appreciate the support by Mr. Kita, Mr. Okazawa, Mr. Kawagishi, Mr. Kido and Mr. Shiotani, Graduate School of Systems Engineering, Wakayama University, and Mr. Kitagawa and Mr. Nakatani of Hitachi Zosen Corporation.

\section{References}

Fujigaki, M., Matui, T., Morimoto, Y., Kita, T., Nakatani, M. \& Kitagawa, A., (2005) Development of Real-Time Displacement Measurement System Using PhaseShifting Digital Holography, Proceedings of the 5th International Conference on Mechanics of Time Dependent Materials (MTDM05), pp. 160-163. Sep. 2005

Fujigaki, M., Kita, T., Okazawa, S., Matui, T. \& Morimoto, Y. (2006) Calibration method with reference plane for phase-shifting digital holographic interferometry using spherical wave. Int. Symp. Adv. Fluid/Sol. Sci. Technol. Exp. Mech., Sapporo, Japan, pp. 11-14.

Hayashi, T., Ugo, R. \& Morimoto, Y. (1986). Experimental observation of stress waves propagating in laminated composites, Experimental Mechanics, Vol. 26, No. 2, pp. 169-174, ISSN 1741-2765

Kolenovic, E., Osten, W., Klattenhoff, R., Lai, S., Von Kopylow, C. and Jüptner, W. (2003) Miniaturized digital holography sensor for distal three-dimensional endoscopy. Appl. Opt. 42, pp. 5167-5172, ISSN 0003-6935, doi:10.1364/AO.42.005167

Morimoto, Y., Nomura, T., Fujigaki, M \& Takahashi, I., (2004). "Reduction of speckle noise effect by divided holograms in phase-shifting digital holography", Proc. of 12th Inter. Conf. on Experimental Mechanics (CD-ROM), Politecnico di Bari, Italy, 29 August-2 Sept..

Morimoto, Y., Nomura, T., Fujigaki, M., Yoneyama, S. and Takahashi, I. (2005a) Deformation measurement by phase-shifting digital holography. Exp. Mech. Vol. 45, No. 1, pp. 65-70, ISSN: 0014-4851, DOI: 10.1007/BF02428991

Morimoto, Y., Matui, T.,. Fujigaki, M \& Kawagishi, N., (2005b) Accurate displacement measurement by windowed holograms in digital holographic interferometry, Proc. of 2005 SEM Annual Conference E Exposition on Experimental Mechanics (CD-ROM), Portland, Oregon, USA, June 2005, pp. 7-9,.

Morimoto, Y., Matui, T., Fujigaki, M. \& Kawagishi, N. (2005c), Effect of weight in averaging of phases on accuracy in windowed digital holographic interferometry for pico- 
meter displacement measurement, Proc. of SPIE, Vol. 6049: Optomechatronic Sensors and Instrumentation, Yasuhiro Takaya, Ed, Dec. 2005

Morimoto, Y., Matui, T., Fujigaki, M. and Kawagishi, N. (2007) Subnano-meter displacement measurement by averaging of phase-difference in windowed digital holographic interferometry,. Opt. Eng. Vol. 46, No. 2,, 025603. ISSN 0091-3286

Morimoto, Y., Matui, T., Fujigaki, M. and Matsui, A., (2008a). Three-dimensional Displacement Analysis by Windowed Phase-shifting Digital Holographic Interferometry, Strain, Vol. 44, No. 1, 49-56

Morimoto, Y. Matui, T, Fujigaki, M. \& Kawagishi, N., (2008b) Effects of Window Size on Accuracy and Spatial Resolution in Windowed Phase-Shifting Digital Holographic Interferometry, Strain (2008) 44, 366-373.

Morimoto, Y. Matui, T, \& Fujigaki, M. (2008c), Application of Three-Dimensional Displacement and Strain Distribution Measurement by Windowed Phase-Shifting Digital Holographic Interferometry, Alan K.T. Lau, J. Lu, Vijay K. Varadan, F.K. Chang, J.P. Tu and P.M. Lam (Eds), Multi-functional Materials and Structures, Advanced Materials Research, Vol.47-50, pp1262-1265, (2008) Trans Tech Publications, Switzerland, doi: 10. 4028, http:/ / www.scientific.net/AMR.47-50.1262

Okazawa, S., Fujigaki, M., Morimoto, Y. \& Matui, T., (2006) Simultaneous Measurement of Out-of-Plane and In-Plane Displacements by Phase-Shifting Digital Holographic Interferometry, Applied Mechanics and Materials (Volumes 3 - 4), Advances in Experimental Mechanics IV, J.M. Dulieu-Barton and S. Quinn (Eds.), pp. 223-228, 10.4028/www.scientific.net/AMM.3-4.223, August, 2006

Pedrini G., Fröning P., Fessler H., \& Tiziani H.J. (1998). In-Line Digital Holographic Interferometry. Appl. Opt., Vol. 37, No. 26, pp. 6262-6269, ISSN 1559-128X, 21553165. doi:10.1364/ AO.37.006262

Pryputniewicz, R. J., (2008), Chap. 24 Holography, In: Springer Handbook of Experimental Solid Mechanics, Sharpe, W. N. Jr., (Ed.), pp. 675-700, Springer, ISBN: 978-0-387-34362-4, Verlag: Springer, US

Ranson, W. F., Sutton, M. A. \& Peters, W. H. (1993). Chap. 8 Holographic and laser speckle interferometry," In: Handbook on Experimental Mechanics, 2nd ed., A.S. Kobayashi, (Ed.), pp. 365-405, VCH Publishers, ISBN: 978-0-471-18864-3.

Schnars, U. \& Ju"ptner, W. (2005) Digital Holography. Springer, ISBN: 978-3-540-21934-7, Berlin.

Sharpe, W. N. Jr., (Ed.), (2008), Springer Handbook of Experimental Solid Mechanics, Verlag: Springer, ISBN: 978-0-387-34362-4, US

Shiotani, K., Fujigaki, M., Matui, T. and Morimoto, Y., (2008) Strain Measurement Method for Tilted Planar Object by Phase-Shifting Digital Holography Combined with Grating Projection Method, Journal of JSEM, Vol. 8, Special Issue, pp. 108-113.

Valery, P. S., Vladimir, S. P., Sergey, A. N., Vitaly, V.B., Igor, N. O. \& Mikhail, M. B. (1996). Strain and Stress Analysis by Holographic and Speckle Interferometry, John Wiley \& Sons, ISBN 0471960772

Yamaguchi, I. \& Zhang, T. (1997), Phase-shifting digital holography, Optics Letters, Vol. 22, No.16, pp. 1268-1270, ISSN 0146-9592 
Zhang, T. and Yamaguchi, I. (1998) Three-dimensional microscopy with phase-shifting digital holography, Optics Letters, Vol. 23, Issue 15, pp. 1221-1223, ISSN 0146-9592, doi:10.1364/OL.23.001221

Yaroslavskii, L. P. \& Merzlyakov, N. S. (1980) Methods of Digital Holography, Consultants Bureau, ISBN 03061096389780306109638 , New York 
ADVANCED HOLOGRAPHY METROLOGY AND IMAGING

Edace by trabele Napdanowa

\author{
Advanced Holography - Metrology and Imaging \\ Edited by Dr Izabela Naydenova
}

ISBN 978-953-307-729-1

Hard cover, 374 pages

Publisher InTech

Published online 09, November, 2011

Published in print edition November, 2011

Advanced Holography - Metrology and Imaging covers digital holographic microscopy and interferometry, including interferometry in the infra red. Other topics include synthetic imaging, the use of reflective spatial light modulators for writing dynamic holograms and image display using holographic screens. Holography is discussed as a vehicle for artistic expression and the use of software for the acquisition of skills in optics and holography is also presented. Each chapter provides a comprehensive introduction to a specific topic, with a survey of developments to date.

\section{How to reference}

In order to correctly reference this scholarly work, feel free to copy and paste the following:

Yoshiharu Morimoto and Motoharu Fujigaki (2011). Three-Dimensional Displacement and Strain Measurements by Windowed Phase-Shifting Digital Holographic Interferometry, Advanced Holography Metrology and Imaging, Dr Izabela Naydenova (Ed.), ISBN: 978-953-307-729-1, InTech, Available from: http://www.intechopen.com/books/advanced-holography-metrology-and-imaging/three-dimensionaldisplacement-and-strain-measurements-by-windowed-phase-shifting-digital-holographi

\section{INTECH}

open science | open minds

\section{InTech Europe}

University Campus STeP Ri Slavka Krautzeka 83/A 51000 Rijeka, Croatia Phone: +385 (51) 770447 Fax: +385 (51) 686166 www.intechopen.com

\section{InTech China}

Unit 405, Office Block, Hotel Equatorial Shanghai No.65, Yan An Road (West), Shanghai, 200040, China 中国上海市延安西路65号上海国际贵都大饭店办公楼 405 单元 Phone: $+86-21-62489820$

Fax: $+86-21-62489821$ 
(C) 2011 The Author(s). Licensee IntechOpen. This is an open access article distributed under the terms of the Creative Commons Attribution 3.0 License, which permits unrestricted use, distribution, and reproduction in any medium, provided the original work is properly cited. 